\title{
DOL SYSTEMS WITH RANK
}

\author{
A. EHRENEEUCHT \\ Department of Computer Science \\ University of Colonado at Boulder, Boulder, \\ Colorado, U.S.A. \\ G. ROZENBERG \\ Department of Mathematics, Utrecht University, \\ Utrecht, Holland \\ and \\ Department of Mathematics, Antwerp University, \\ UIA, Wilrijk, Belgium.
}

INTRODUCTION

L systems (also called Lindenmayer systems or developmental systems) have recently gained considerable attention in both formal language theory and theoretical biology (see, e.g., [45], [60], [66] and their references). Among the developmental systems which are under active investigation now are the so called DOL systems (see, e.g., [14], [45], [75], [82], [83] and [95]). One of the most interesting and physically best motivated topics in the theory of I systems is that of "local versus global properties". It is concerned with explaining on the local level (sets of productions) global properties (i.e., those properties of the language on of the sequence generated by an $L$ system whose formulation is independent on the I system itself). Examples of papers in this direction are [95] and [116].

This paper is concemed with the topic of "Iocal versus global properties" in the case of DoL systems. It provides structural characterization of those DOL systems whose growth functions (see, e.g., [98] and [111]) are polynomially bounded. 
We use standard formal-language theoretical terminology and notation. (Perhaps the only unusual notation is "\# $(x)$ " meaning "the number of occurrences of the letter a in the word $x^{\prime \prime}$. )

1. DOL SYSTEMS, SEQUENCES AND LANGUAGES

In this section we introduce basic definitions and terminology concerning DoL systems.

Definition 1. A deterministic L system without interactions, abbreviated DoL system, is a triple $G=\langle\Sigma, \delta, \omega\rangle$ where $\Sigma$ is a finite nonempty set (the alphabet of $G$ ), $w$ is a nonempty word over $\Sigma$ (the axiom of $G$ ) and $\delta$ (the transition function of $G$ ) is a homomorphism from $\Sigma$ into $\Sigma^{*}$. $G$ is called a propagating DoL system, abbreviated PDOL system, if $\delta$ is a homomorphism from $\Sigma$ into $\Sigma^{+}$.

Definition 2. Let $G=\langle\Sigma, \delta, \omega\rangle$ be a DoL system and let, for $i \geqslant 0, \delta^{i}$ denote the i-folded composition of $\delta$ (with $\delta^{0}$ being the identity function on $\Sigma^{*}$ ). The sequence of $G$, denoted $\&(G)$, is the sequence $\omega_{0}, \omega_{1}, \ldots$ of words over $\Sigma^{*}$ such that $\omega_{0}=\omega$ and $\omega_{i}=\delta^{i}(\omega)$ for every $i \geqslant 0$. The language of $G$, denoted $\mathcal{L}(G)$, is defined by $\mathcal{L}(G)=$ $\left\{x \in \Sigma^{*}: \delta^{i}(\omega)=x\right.$ for some $\left.i \geqslant 0\right\}$. A letter a from $\Sigma$ is called use$\underline{\text { ful (in } G)}$ if a occurs in $\delta^{i}(w)$ for some $i \geqslant 0$.

Definition 3 . Let $K$ be a language (a sequence of words). $K$ is called a DOL or a PDOL language (sequence) if for some DoL system $G$ or for some PDOL system $G$ respectively $\mathcal{L}(G)=K(\mathscr{E}(G)=K)$.

Definition 4. Let $G=\langle\Sigma, \delta, \omega\rangle$ be a DOL system and a be in $\Sigma$. We say that a is limited (in G) if there exists a constant $C$ such that, for every $i \geqslant 0,\left|\delta^{i}(a)\right|<c$. 
Definition 5. Let $G=\langle\Sigma, \delta, w\rangle$ be a DoL system. We say that $G$ is polynomially bounded if there exists a polynomial $p$ such that, for all $i \geqslant 0,\left|\delta^{i}(\omega)\right| \leqslant p(i)$.

Example 1. Let $G=\langle\Sigma, \delta, \omega\rangle$ where $\Sigma=\{a\}, \omega=a$ and $\delta$ is such that $\delta(a)=a^{2}$. Then $G$ is a PDOL system, $\&(G)=a, a^{2}, a^{4}, \ldots, \mathscr{L}(G)=$ $\left\{a^{2^{i}}: i \geqslant 0\right\}$ and $G$ is not a polynomially bounded system.

Example 2. Let $G=\langle\Sigma, \delta, \omega\rangle$ where $\Sigma=\{a, b, c, d\}, \omega=c a b$ and $\delta$ is such that $\delta(a)=c a b, \delta(b)=b, \delta(c)=\Lambda$ and $\delta(d)=d$. Then $G$ is $a$ DOL system (but it is not a PDOL system), $\&(G)=c a b, c a b^{2}, c a b^{3}, \ldots$, $\mathscr{L}(G)=\left\{\mathrm{cab}^{\mathrm{n}}: \mathrm{n} \geqslant 1\right\}$ and $G$ is polynomialiy bounded. The letters $\mathrm{b}$ and $c$ are limited but the letter a is not limited. The letter d is not useful.

2. DOL SYSTEMS WITH RANK

In this section basic notions concerning DoL systems with rank (the subject of investigation of this paper) are introduced.

Definition 6 . Let $G=\langle\Sigma, \delta, \omega\rangle$ be a DOL system. The rank of a letter $a$ in $G$, denoted $\rho_{G}(a)$, is defined inductively as follows: (i) If a is limited in $G$, then $\rho_{G}(a)=1$.

(ii) Let $\Sigma_{0}=\Sigma$ and $\delta_{0}=\delta$. Let, for $j \geqslant 1, \delta_{j}$ denote the restriction of $\delta$ to $\Sigma_{j}=\Sigma-\left\{a: \rho_{G}(a) \leqslant j\right\}$. For $j \geqslant 1$, if $a$ is linited in $\left\langle\Sigma, \delta_{j}, a\right\rangle$ then $\rho_{G}(a)=j+1$.

Definition 7. Let $G$ be a DOL system. We say that $G$ is a DOL system with rank if every letter which is useful in $G$ has a rank. If $G$ is a DOL system with rank then the rank of $G$ is defined as the largest of the ranks of letters useful in $G$. 
Example 3 . Let $G$ be the DoL system from Example 1. Then the letter $a$ has no rank and consequently $G$ is not a DoL system with rank.

Example 4. Let $G$ be the DOL system from Example 2. Then $\rho_{G}(b)=\rho_{G}(c)=\rho_{G}(d)=1$ and $\rho_{G}(a)=2$. Consequent $1 y$ is a DOL system with rank and the rank of $G$ equals 2 .

3. RESULTS

In this section main results concerning DoL systems with rank are stated.

First of all it turns out that the notion of a DOL system with rank is an effective one in the following sense.

Theorem 1. It is decidable whether an arbitrany DoL system is a DOL system with rank.

The language (on sequence) equivalence problem for DoL systems (i.e., whether two arbitrary DOL systems produce the same language or sequence) is one of the most intriguing and the longest open problems in the theory of $L$ systems. At the time of writing of this paper we were not able to settle this question for arbitrary DoL systems with rank. However for propagating DOL systems with rank we have the following result.

Theorem 2. There exists an algorithm which given two arbitrary PDOL systems with rank $G_{1}$ and $G_{2}$ will decide whether on not $\mathcal{L}\left(G_{1}\right)=$ $\mathscr{L}\left(G_{2}\right) \quad\left(\mathscr{E}\left(G_{1}\right)=\mathscr{E}\left(G_{2}\right)\right)$.

Thus PDOL systems with rank constitute at the present time the largest nontrivial subclass of the class of DoL systems for which the language (and sequence) equivalence problem is decidable. 
One of the interesting features of DOL systems with rank is that the sequence of Parikh vectors corresponding to a DoL system with rank always has an ultimately periodic polynomial description in the following sense.

Theorem 3 . Let $G=\langle\Sigma, \delta, \omega\rangle$ be a DoL system with rank. For every letter $a$ which is useful in $G$ there exist integers $s, r$ and $a$ sequence $P_{0}, P_{1}, \ldots, P_{n-1}$ of polynomials (with rational coefficients and with positive leading coefficients) such that, for every $j>s$, $\#_{a}(\delta(i r+t)(\omega))=p_{t}(i)$, where $j=$ irtt and $0 \leqslant t<r$.

The notion of a DOL system with rank is an important one as it provides the structural characterization of polynomially bounded DOL systens.

Theorem 4. A DOL system is polynomially bounded if and only if it is a DOL system with rank.

In fact the rank of a Dot system characterizes quite precisely the growth of a polynomially bounded DOL system, as is shown by the following result.

Theorem 5. If $G=\langle\Sigma, \delta, \omega\rangle$ is a DoL system, $a$ is in $\Sigma$ and $n$ is a nonnegative integer, then $\rho_{G}(a)=n+1$ if and only if there exist polynomials $p_{a}(i), q_{a}(i)$ of degree $n$, such that, for all $i \geqslant 0$, $p_{a}(i) \leqslant\left|\delta^{i}(a)\right| \leqslant q_{a}(i)$. Moreover one car, by exploiting the structural properties of $G$, effectively construct polynomials $p_{a}(i)$ and $q_{a}(i)$ with this property.

As corollaries of Theorem 4 we get a number of results concerning polynomially bounded DoL systems. Fon example, we have the following.

Corollary 1. There exists an algorithm which given two arbitrary polynomially bounded PDOL systems $G_{1}$ and $G_{2}$ will decide whether 
or $\operatorname{not} \mathscr{L}\left(G_{1}\right)=\mathscr{L}\left(G_{2}\right) \quad\left(\mathscr{E}\left(G_{1}\right)=\mathscr{E}\left(G_{2}\right)\right)$.

Corollary 2. If $G=\langle\Sigma, \delta, \omega\rangle$ is a polynomially bounded DoL system, then there exist integers $s, r$ and a sequence $p_{0}, p_{1}, \ldots, p_{r-1}$ of polynomials (with rational coefficients and with positive leading coefficient) such that, for every $j>s,\left|\delta^{(i n+t)}(\omega)\right|=p_{t}(i)$, where $j=$ irtt and $0 \leqslant t<r$. 